Results The total number of respondents was 57, with $65 \%$ of questionnaires $(n=38)$ completed. Responses came from 17 different hospitals and 18 different graduation countries, 52\% $(n=30)$ non EU. 28\% $(n=13)$ worked at SHO level and the remainder as registrars. 57\% $(n=33)$ were eligible for training schemes. $55 \%(\mathrm{n}=32)$ did not have a supervisor or named trainer. $86 \%$ had the opportunity to avail of internal CPD points and $83 \%$ for external, with $83 \%$ able to complete an audit every year. Only 34\% (n-28) were given an opportunity to undertake research each year, $68 \%$ are planning to stay in Ireland long-term. Open ended answers indicated that doctors don't like being excluded from training and it causes them to either switch to GP or leave Ireland altogether to complete their paediatric training.

Conclusion The majority of doctors who are not on a BST/ HST scheme have the opportunity to perform an audit yearly and to get the required internal/external CPD points. Access to a named trainer/mentor was patchy and those eligible for training schemes have a more positive response and tend to have a much higher preference to stay in Ireland long-term than non-eligible doctors.

With EWTD pressures we may need to consider more sustainable ways of recruiting and retaining this vital workforce.

\section{P262 ORGANISATIONAL AND INDIVIDUAL TRAINEE LEARNING FROM INCIDENTS AND ADVERSE EVENTS}

Natalie Bee, Anne Dokubo*, Sarah Berwick, Sonia Joseph. Royal Hospital for Sick Children, Edinburgh, UK

\subsection{6/archdischild-2019-epa.612}

Background Trainee surveys and feedback to trainee representatives suggest that few trainees have gained feedback on systems outcomes from Datix (adverse event reporting forms) or Significant Event Analyses.

Thus creating a barrier to systems learning, personal reflection and empowerment that trainees can influence and improve the system in their healthcare setting.

Aim To develop a learning adaptive system that utilises current infrastructures and innovation, where required; in order to improve trainee learning and feedback from DATIX, significant event analysis and paediatric emergency team calls.

Methods Learning outcomes from anonymised DATIX reports and completed Significant Event Analysis Reviews were studied and presented quarterly at the paediatric trainee regional teaching with a key focus on non-technical skills, systems changes and limiting risk.

Paediatric emergency team calls are infrequent in paediatrics and therefore these are presented less frequently on a 6 monthly basis in conjunction with the resuscitation officer.

Results Through working closely with management and senior nursing staff it has been possible to increase trainee feedback whilst encompassing the RCPCH Quality Improvement Progress Learning Outcomes.

A survey of the eleven trainees attending the most recent feedback session unanimously agreed/strongly agreed that these sessions have been very useful for their training and development in patient safety and management within healthcare. All trainees agreed that this was useful learning within the regional teaching programme which ensures attendance.

Conclusions At times, trainees have described feeling like passengers or passive recipients of 'the system' they work in. Our small initiative is the first step in challenging the current status quo and changing the narrative around trainee engagement within the NHS. It was low cost but had a positive impact on perception. Key next stages are to embed this feedback and training for other groups of staff and to build an infrastructure linked to clinical management teams that enables true organisational learning.

\section{P263 INVESTIGATION OF THE FIRST AFEBRILE SEIZURE IN THE PAEDIATRIC ED: A SYSTEMATIC REVIEW}

${ }^{1}$ Haaris Aziz Shiwani, ${ }^{1}$ Eoin MacMeanmain, ${ }^{1}$ Evan Hurley-O'Dwyer, ${ }^{1}$ Grace Rothwell-Kelly, ${ }^{1}$ Nicholas Arrotta, ${ }^{1}$ Louise Sweeney, ${ }^{2}$ Danyal Memon*, ${ }^{3}$ Shaheer Aziz, ${ }^{1}$ Mohammad Danish Yusuf, ${ }^{1}$ Hadeer Ameen, ${ }^{4}$ Eleanor Molloy. ${ }^{1}$ School of Medicine, Trinity College Dublin, Dublin, Ireland; ${ }^{2}$ Royal Victoria Hospital, Belfast, UK; ${ }^{3}$ Jessenius Faculty of Medicine, Martin, Slovakia; ${ }^{4}$ Department of Paediatrics, School of Medicine, Trinity College Dublin, Dublin, Ireland

\subsection{6/archdischild-2019-epa.613}

Background There is currently no consensus on investigations required in the paediatric emergency department following a first afebrile seizure. This systematic review aims to compare investigations commonly used and to evaluate their effectiveness and diagnostic value.

Methods In this systematic review keywords such as 'afebrile', 'seizure', 'paediatric' and 'emergency department' were searched for in numerous databases (SCOPUS, MEDLINE, ProQuest, EMBASE, CINAHL, Cochrane and Grey Literature). The resulting titles and abstracts were manually reviewed to exclude irrelevant articles. Finally, the studies that met our inclusion and exclusion criteria were selected to undergo further analysis.

Results A total of eleven articles of the initial 9946 were analysed. Five of these evaluated blood tests $(n=303)$ including full blood count, sodium, potassium, calcium, glucose and leucocytes with no consistent findings. Seven studies evaluated CT or MRI $(n=1208)$. Cumulatively, abnormal results were present in $12.3 \%$ of these neuroimaging studies. EEG was also performed in three of the seven studies that performed neuroimaging $(n=420)$ with abnormalities in $37.1 \%$ of these.

Discussion In patients presenting to the paediatric emergency department with a first afebrile seizure, thorough history and examination are essential. In unison with the American Academy of Neurology's guidelines, EEG proves to be the most valuable investigation to perform. Neuroimaging may be warranted in some cases to exclude underlying CNS pathology or if structural abnormalities are suspected. Laboratory studies are a necessary adjunct but have limited diagnostic value.

\section{P264 IMPACT OF HOMELESSNESS ON CHILDREN PRESENTING TO A TERTIARY EMERGENCY DEPARTMENT}

${ }^{1}$ Ann-Marie Hayes*, ${ }^{2}$ Briggita Joyce, ${ }^{3}$ Roisin McNamara, ${ }^{4}$ kke Okafor. ${ }^{1}$ Temple St Children's University Hospital, Dublin, Ireland; 'Temple Street Children's University Hospital, Dublin, Ireland; ${ }^{3}$ Temple Street Children's University Hospital, Dublin, Ireland; ${ }^{4}$ Temple Street Children's University Hospital, Dublin Ireland, Ireland

\subsection{6/archdischild-2019-epa.614}

Introduction Of 53,000 children (aged $0-16$ years) seen in the Emergency Department (ED) of Temple Street Children's University Hospital (TSCUH), a tertiary Paediatric hospital in 